\title{
Comparison of renal region, cerebral and peripheral oxygenation for predicting postoperative renal impairment after CABG
}

\author{
Ilonka N. de Keijzer ${ }^{1}$ (D ) Marieke Poterman ${ }^{1}$ (D) A Anthony R. Absalom ${ }^{1}$ (D) Jaap Jan $\operatorname{Vos}^{1}$ (D) $\cdot$ Massimo A. Mariani $^{2}$. \\ Thomas W. L. Scheeren ${ }^{1}$ (D)
}

Received: 7 December 2020 / Accepted: 31 March 2021 / Published online: 20 April 2021

(c) The Author(s) 2021

\begin{abstract}
Patients undergoing coronary artery bypass grafting (CABG) are at risk of developing postoperative renal impairment, amongst others caused by renal ischemia and hypoxia. Intra-operative monitoring of renal region tissue oxygenation $\left(\mathrm{SrtO}_{2}\right)$ might be a useful tool to detect renal hypoxia and predict postoperative renal impairment. Therefore, the aim of this study was to assess the ability of intra-operative $\mathrm{SrtO}_{2}$ to predict postoperative renal impairment, defined as an increase of serum creatinine concentrations of $>10 \%$ from individual baseline, and compare this with the predictive abilities of peripheral and cerebral tissue oxygenation $\left(\mathrm{SptO}_{2}\right.$ and $\mathrm{SctO}_{2}$, respectively) and renal specific tissue deoxygenation. Forty-one patients undergoing elective CABG were included. Near-infrared spectroscopy (NIRS) was used to measure renal region, peripheral (thenar muscle) and cerebral tissue oxygenation during surgery. Renal region specific tissue deoxygenation was defined as a proportionally larger decrease in $\mathrm{SrtO}_{2}$ than $\mathrm{SptO}_{2}$. ROC analyses were used to compare predictive abilities. We did not observe an association between tissue oxygenation measured in the renal region and cerebral oxygenation and postoperative renal impairment in this small retrospective study. In contrast, $\mathrm{SptO}_{2}$ decrease $>10 \%$ from baseline was a reasonable predictor with an AUROC of 0.767 (95\% CI 0.619 to $0.14 ; \mathrm{p}=0.010$ ). Tissue oxygenation of the renal region, although non-invasively and continuously available, cannot be used in adults to predict postoperative renal impairment after CABG. Instead, peripheral tissue deoxygenation was able to predict postoperative renal impairment, suggesting that $\mathrm{SptO}_{2}$ provides a better indication of 'general' tissue oxygenation status.
\end{abstract}

Registered at ClinicalTrials.gov: NCT01347827, first submitted April 27, 2011.

Keywords Coronary artery bypass grafting $(\mathrm{CABG}) \cdot$ Renal oxygenation $\cdot$ Cerebral oxygenation $\cdot$ Acute kidney injury . Postoperative renal impairment

\section{Introduction}

Cardiac surgery is often complicated by acute kidney injury (AKI). The incidence of AKI after cardiac surgery, as identified by commonly used AKI classification systems (AKIN, KDIGO, or RIFLE) varies from 5 to $42 \%$ depending on comorbidity, surgical technique, and the population studied $[1,2]$. Even minor renal impairment—not identified by these

Ilonka N. de Keijzer

i.n.de.keijzer@umcg.nl

1 Department of Anaesthesiology, University Medical Centre Groningen, Hanzeplein 1, Groningen 9713 GZ, The Netherlands

2 Department of Cardiothoracic Surgery, University Medical Centre Groningen, Groningen, The Netherlands
AKI classifications-is associated with increased postoperative morbidity and mortality after cardiac surgery $[3,4]$. In the time since the AKI classifications have become widely accepted, studies have been published that suggest that smaller changes in serum creatinine should not be ignored $[5,6]$. Therefore it has been advocated that impaired postoperative renal function is best evaluated as an increase of postoperative serum creatinine levels compared to individual preoperative levels $[4,7]$.

It remains difficult to properly predict who is at risk for developing postoperative renal impairment after cardiac surgery, especially when cardiopulmonary bypass (CPB) is used, as it can cause haemodynamic alterations and possibly disturb renal autoregulation [8]. Intra-operative tissue oxygenation measured in the renal region $\left(\mathrm{SrtO}_{2}\right)$ offers a non-invasive dynamic insight into tissue oxygenation, by 
assessing the balance between oxygen delivery and oxygen consumption at the tissue level [9], and may serve to identify patients at risk of renal function loss. $\mathrm{SrtO}_{2}$ was correlated to renal venous oxygen saturation $\left(r_{r m}=0.61\right.$, $\mathrm{p}<0.001$ ) in adult on-pump cardiac surgery patients [10] and in paediatric patients renal tissue deoxygenation has been shown to be associated with adverse postoperative outcomes [11-15]. However, whether $\mathrm{SrtO}_{2}$ also predicts postoperative impairment of renal function in adult cardiac surgery patients, is unknown.

In the current study we hypothesized that tissue deoxygenation of the renal region during coronary artery bypass grafting (CABG) is a good predictor of postoperative renal impairment in adult patients with normal preoperative serum creatinine concentrations. Additionally, the ability of tissue deoxygenation of the renal region to predict postoperative renal impairment was compared with that of peripheral and cerebral tissue oxygenation $\left(\mathrm{SptO}_{2}\right.$ and $\mathrm{SctO}_{2}$, respectively) and renal region specific tissue deoxygenation (defined as a proportionally larger decrease in $\mathrm{SrtO}_{2}$ than $\mathrm{SptO}_{2}$ ).

\section{Material and methods}

This study is a secondary analysis of data from a randomized controlled trial comparing the incidence and severity of cerebral oxygen desaturation during CABG, with and without CPB [16]. Data were obtained in 59 patients who were randomized to on-pump or off-pump CABG and who had near-infrared spectroscopy (NIRS) sensors applied at the flank, the thenar muscle, and the forehead to measure renal region, peripheral, and cerebral tissue oxygenation, respectively. The study was performed at the University Medical Centre Groningen, The Netherlands, between June 2011 and May 2012. Ethical approval (Reference: METc2011/045) was obtained from the institutional ethical committee, Groningen, The Netherlands. The study was registered at ClinicalTrials.gov (NCT01347827).

\subsection{Study procedures and patient selection}

The details of the study protocol and intra-operative anaesthetic management have been published previously [16]. In brief, patients aged 18 years and older were included when both the operating surgeon and the responsible anaesthesiologist considered the patient eligible for CABG performed either with or without CPB. Exclusion criteria included pre-existing acute or chronic renal impairment (serum creatinine concentration above $200 \mu \mathrm{mol} \cdot \mathrm{l}^{-1}$ ).

\subsection{Determination of renal impairment}

We defined renal impairment as an increase of serum creatinine concentrations of $>10 \%$ from individual baseline values within the first 7 days postoperatively.

\subsection{Oxygenation measurement}

During CABG, tissue oxygenation was measured noninvasively and continuously by NIRS with the INVOS 5100 Oximeter (Covidien, Dublin, Ireland (now part of Medtronic, Minneapolis, Minnesota (USA)). After induction of anaesthesia, an ultrasound examination of the left and right superior lumbar region was performed by the anaesthesiologist to determine the location of the kidneys and their depth beneath the skin surface. Assessment of renal tissue oxygenation was only considered feasible in patients where the kidney surface was located less than $5 \mathrm{~cm}$ from skin level. Sensors were applied to the skin of the superior lumbar regions to assess $\mathrm{SrtO}_{2}$ of both kidneys separately. Cerebral oxygenation was monitored using NIRS monitors and sensors from two manufacturers, but for this study we only used the oxygenation measurements from the INVOS Cerebral Oximeter 5100C (Covidien, Dublin, Ireland (now part of Medtronic, Minneapolis, Minnesota (USA)). Another sensor was placed on the right thenar eminence to measure peripheral tissue oxygenation with an InSpectra ${ }^{\mathrm{TM}}$ device (Hutchinson Technology, Hutchinson, USA).

\subsection{Calculation of oxygenation data}

Baseline renal region, cerebral and peripheral tissue oxygenation data were obtained after induction of anaesthesia with the lungs of the patient being ventilated with an inspiratory oxygen fraction $\left(\mathrm{FiO}_{2}\right)$ of 0.4 and were calculated as the mean value of $\mathrm{SrtO}_{2}, \mathrm{SctO}_{2}$ and $\mathrm{SptO}_{2}$ during the $5 \mathrm{~min}$ before surgical incision. The area under the threshold (AUT) of renal region and cerebral tissue deoxygenation was quantified for absolute and relative thresholds of $\mathrm{SrtO}_{2}$ and $\mathrm{SctO}_{2}$ in \% min. For absolute values we applied thresholds of $90 \%, 80 \%, 70 \%$, and $60 \%$. For relative changes, we calculated AUTs for thresholds that were $5 \%, 10 \%$, and $15 \%$ below each individual's baseline. For $\mathrm{SptO}_{2}$ only the AUT for a relative threshold of $>10 \%$ decrease from baseline $\mathrm{SptO}_{2}$ was assessed.

Oxygenation data below each threshold were expressed as the AUT (in \% min) below each threshold, and were calculated using the following formulae: 
- for absolute thresholds: AUT $<90 \%=(90-$ current $\left.\mathrm{SrtO}_{2}\right) \times$ time (minutes)

- for relative thresholds: AUT $>5 \%$ below baseline $\mathrm{SrtO}_{2}=\left(0.95 \times\right.$ baseline $\mathrm{SrtO}_{2}-$ current $\left.\mathrm{SrtO}_{2}\right) \times$ time (minutes).

The AUT of renal region specific tissue deoxygenation was calculated as follows: individual baseline $\mathrm{SrtO}_{2}$ and $\mathrm{SptO}_{2}$ were set at $100 \%$. All intraoperative values were calculated as a percentage change from baseline. Subsequently, $\mathrm{SrtO}_{2}$ values (in \% from baseline $\mathrm{SrtO}_{2}$ ) were subtracted from $\mathrm{SptO}_{2}$ (in \% from baseline $\mathrm{SptO}_{2}$ ) and all positive values (when $\mathrm{SptO}_{2}>\mathrm{SrtO}_{2}$ ) were summed up to assess the AUT of renal region specific tissue deoxygenation (Fig. 1).

\subsection{Statistical analysis}

Statistical analyses were performed in SPSS version 23 (IBM Inc., Chicago, USA). Normality of continuous variables was assessed by the Shapiro-Wilk test. Continuous data are expressed as mean (SD) for parametric data or as median [IQR range] for non-parametric data. To compare continuous variables for parametric and nonparametric data, an independent $T$ test or Mann-Whitney $U$ test was performed respectively. Categorical variables are presented as number (proportion) and compared by using Fisher's Exact or Chi-square test, depending on the number of patients per group. Correlation between left and right renal region tissue oxygenation measurements was assessed using Spearman's correlation coefficient. Receiver operating characteristics (ROC) curves were created to assess the predictive value of absolute and relative oxygenation thresholds. Statistical significance was defined as $\mathrm{p}<0.05$.

\section{Results}

\subsection{Patient characteristics}

In the original study 59 patients were included of whom 18 did not have sufficient data for this secondary analysis. Reasons for exclusion were: no renal region tissue oxygenation data $(n=12)$, no peripheral tissue oxygenation data $(n=1)$, or insufficient renal region and peripheral tissue oxygenation data $(n=5)$. Renal region tissue oxygenation data were missing due to depth or the location of the kidneys, e.g. $>5 \mathrm{~cm}$ deep, or the kidney(s) could not be visualized on ultrasound imaging, and for peripheral tissue oxygenation NIRS sensors were not applied. Thus, 41 patients with a mean age of 63 (9) years remained for analysis: 19 patients who had been randomized to undergo $\mathrm{CABG}$ with $\mathrm{CPB}$ and 22 patients randomized to undergo $\mathrm{CABG}$ without $\mathrm{CPB}$ for the purpose of the original study [16]. All patients were classified as ASA physical status III. In 35 out of these 41 patients $\mathrm{SrtO}_{2}$ data were obtained bilaterally, and in these cases left and right $\mathrm{SrtO}_{2}$ values were averaged (mean correlation between left and right kidney of $r=0.80, p<0.001$ ). In 3 out of 41 cases cerebral oxygenation data was obtained bilaterally and the left and right $\mathrm{SctO} 2$ values were averaged. In 11 patients (27\%) serum creatinine concentrations increased $>10 \%$ from baseline within the first 7 days postoperatively. Patients with postoperative renal impairment had a longer duration of surgery (197 [182-240] min vs. 173 (26) $\mathrm{min}, \mathrm{p}=0.008)$ and needed more grafts $(4[3,4]$ vs. $3[3], p=0.009)$. Data describing demographic, intra-operative and postoperative patient characteristics are shown in Table 1.

\subsection{Tissue oxygenation}

Baseline $\mathrm{SrtO}_{2}, \mathrm{SctO}_{2}$ and $\mathrm{SptO}_{2}$ values of patients with and without postoperative renal impairment were similar (Table 2). The AUT below all predefined absolute and
Fig. 1 Example of the calculation of renal region specific tissue deoxygenation. The grey area is the area under the threshold (AUT; $(\% \min )$ ) of renal region specific tissue deoxygenation, which was calculated by subtracting the $\mathrm{SrtO}_{2}$ in $\%$ from baseline (renal tissue (blue line)) from the $\mathrm{SptO}_{2}$ in \% from baseline (peripheral tissue (orange line))

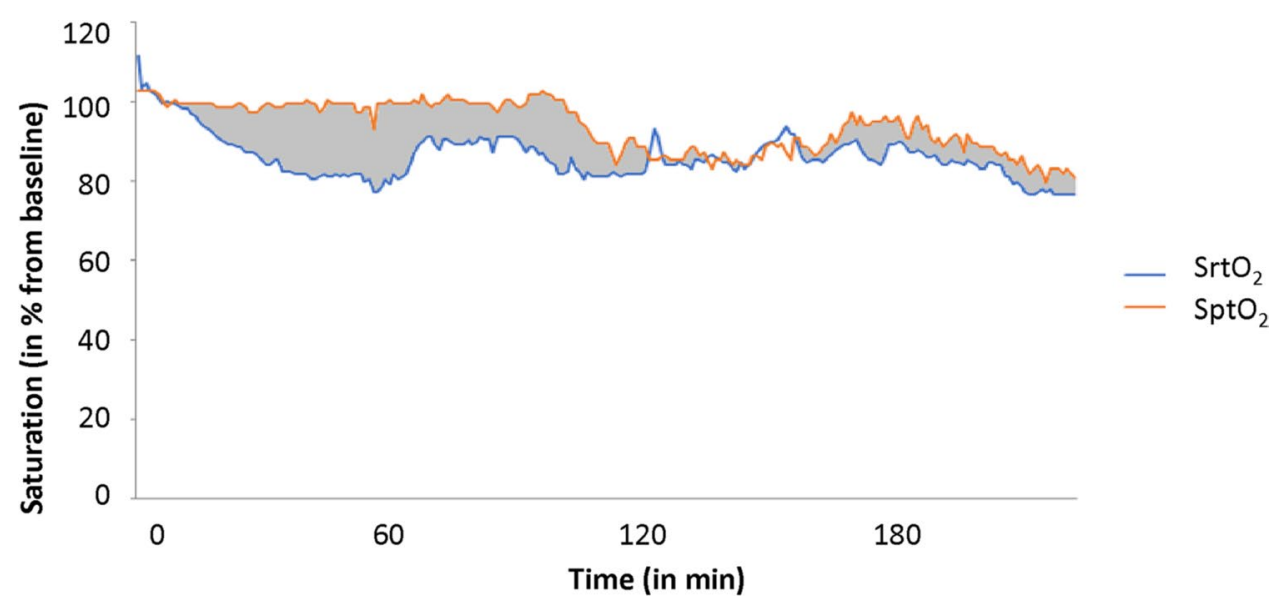


Table 1 Demographic, intra-operative and postoperative characteristics in patients undergoing on-pump or off-pump CABG, compared by postoperative renal function. Variables are number (\%), mean (SD) or median [IQR]

\begin{tabular}{|c|c|c|c|c|}
\hline & All patients $(n=41)$ & $\begin{array}{l}\text { Patients without renal impair- } \\
\text { ment }(\mathrm{n}=30)\end{array}$ & $\begin{array}{l}\text { Patients with renal impair- } \\
\text { ment }(\mathrm{n}=11)\end{array}$ & $P$ value \\
\hline Gender; male & $38(93 \%)$ & $27(90 \%)$ & $11(100 \%)$ & 0.551 \\
\hline Age; years & $63(9)$ & $62(9)$ & $66(9)$ & 0.155 \\
\hline BMI; $\mathrm{kg} \mathrm{m}^{-2}$ & $27.6(3.4)$ & $27.3(3.2)$ & $28.4(4.0)$ & 0.361 \\
\hline Operated on CPB (on-pump) & $19(46 \%)$ & $12(40 \%)$ & $7(64 \%)$ & 0.290 \\
\hline EuroSCORE & $1.8[1.3-3.0]^{1}$ & $1.5[1.0-2.9]$ & $2.6[1.6-3.3]$ & 0.095 \\
\hline DM type II & $10(24 \%)$ & $8(27 \%)$ & $2(18 \%)$ & 0.700 \\
\hline Hypertension & $11(27 \%)$ & $7(23 \%)$ & $7(64 \%)$ & 0.445 \\
\hline Smoking & $26(63 \%)^{2}$ & $19(63 \%)$ & $7(64 \%)$ & 1.000 \\
\hline Pre-operative LVEF & & & & 0.349 \\
\hline $55-70 \%$ & $19(46 \%)$ & $16(53 \%)$ & $3(27 \%)$ & \\
\hline $40-55 \%$ & $19(46 \%)$ & $12(40 \%)$ & $7(64 \%)$ & \\
\hline$<40 \%$ & $3(7 \%)$ & $2(7 \%)$ & $1(9 \%)$ & \\
\hline Preoperative Creatinine; $\mu \mathrm{mol} 1^{-1}$ & $84[75-103]$ & 84 [76-94] & $93(28)$ & 0.942 \\
\hline Baseline $\mathrm{Hb} ; \mathrm{mmol} \mathrm{l}^{-1}$ & $8.3(0.7)$ & $8.4[8.2-8.6]$ & $8.2(0.9)$ & 0.851 \\
\hline Duration of surgery; min & $180[159-196]$ & $173(26)$ & $197[182-240]$ & $0.008^{*}$ \\
\hline CPB time ${ }^{\mathrm{a}} ; \min$ & $83(22)$ & $82(20)$ & $86(26)$ & 0.697 \\
\hline Aorta cross clamp time ${ }^{\mathrm{a}}$; min & $53(14)$ & $53(13)$ & $53(15)$ & 0.997 \\
\hline No. of grafts per patient & $3[3,4]$ & $3[3-3]$ & $4[3,4]$ & $0.009 *$ \\
\hline \multicolumn{5}{|l|}{ Intra-operative analyses } \\
\hline Lactate; highest & $2.4(0.9)$ & $2.4(0.8)$ & $2.5(1.1)$ & 0.883 \\
\hline Mean intraoperative MAP; $\mathrm{mmHg}$ & $70(6)$ & $70(6)$ & $71(7)$ & 0.574 \\
\hline Stay on ICU; days & $1[1-1]$ & $1[1-1]$ & $1[1-4]$ & 0.315 \\
\hline Stay in hospital; days & $8[6-11]$ & $8(3)$ & $11(5)$ & 0.052 \\
\hline
\end{tabular}

${ }^{1}$ EuroSCORE is missing from 2 patients. ${ }^{2}$ Smoking is missing from 1 patient

${ }^{a}$ Only in on-pump patients. BMI body mass index, $C P B$ cardiopulmonary bypass, ASA american society of anesthesiologist status, EuroSCORE European system for cardiac operative risk evaluation, DM type II diabetes mellitus type 2, $L V E F$ left ventricular ejection fraction, $H b$ haemoglobin, MAP mean arterial pressure, $I C U$ intensive care unit. Significance is indicated with $*$ for $\mathrm{p}<0.05$

relative $\mathrm{SrtO}_{2}$ and $\mathrm{SctO}_{2}$ thresholds, and renal specific tissue deoxygenation did not show any significant differences between patients with and without postoperative renal impairment. However, the AUT $\mathrm{SptO}_{2}>10 \%$ decrease from baseline was significantly higher in patients with postoperative renal impairment (215 [72-934] \% min vs. 36 [0-313] \% min, $\mathrm{p}=0.009)$. In 8 patients no deoxygenation of peripheral tissue occurred, i.e. $\mathrm{SptO}_{2}$ did not drop below $90 \%$ from baseline, and none of those patients developed postoperative renal impairment. In a subgroup analysis, tissue oxygenation was compared between on- and offpump CABG. No differences were found between subgroups in baseline $\mathrm{SrtO}_{2}$, $\mathrm{SctO}_{2}$ and $\mathrm{SptO}_{2}$ and all absolute and relative thresholds (Table 3 ). However, the extent of renal region specific tissue deoxygenation was higher in on-pump CABG compared to off-pump CABG (1015 [443-1784] \% min vs. 327 [81-1044] \% min, $\mathrm{p}=0.025)$.

\subsection{ROC analyses}

The AUT $\mathrm{SptO}_{2}>10 \%$ decrease from baseline showed the highest area under the ROC (AUROC) of 0.767 $(95 \% \mathrm{CI}=0.691$ to $0.914 ; \mathrm{p}=0.010$ ) for predicting postoperative renal impairment (Table 4). The optimal cut-off $\mathrm{SptO}_{2}$ was $65 \%$ min with a sensitivity of $91 \%$ and a specificity of $63 \%$. None of the thresholds set for renal region or cerebral tissue deoxygenation was able to predict postoperative renal impairment, nor was renal region specific tissue deoxygenation. Of note, the preoperative creatinine levels were also not predictive of postoperative renal impairment (AUROC of 0.508 (95\% CI 0.334 to $0.864 ; \mathrm{p}=0.941)$. 
Table 2 Intra-operative oxygenation data of patients with and without postoperative renal impairment. Variables are mean $(\mathrm{SD})$ or median $[\mathrm{IQR}]$

\begin{tabular}{|c|c|c|c|}
\hline & No renal impairment $(\mathrm{n}=30)$ & Renal impairment $(\mathrm{n}=11)$ & $\mathrm{P}$ value \\
\hline \multicolumn{4}{|l|}{$\mathrm{SrtO}_{2}$} \\
\hline Baseline; $\%$ & $80[75-86]$ & $77(11)$ & 0.571 \\
\hline \multicolumn{4}{|l|}{ AUT absolute decrease $\mathrm{SrtO}_{2} ; \%$ min } \\
\hline Below $90 \% \mathrm{SrtO}_{2}$ & $2396[1372-3347]$ & $4333(3539)$ & 0.359 \\
\hline Below $80 \% \mathrm{SrtO}_{2}$ & 779 [204-1408] & $876[115-5348]$ & 0.407 \\
\hline Below $70 \% \mathrm{SrtO}_{2}$ & 114 [12-275] & $172[54-1880]$ & 0.287 \\
\hline Below $60 \% \mathrm{SrtO}_{2}$ & $13[0-53]$ & $34[0-285]$ & 0.226 \\
\hline \multicolumn{4}{|l|}{ AUT relative decrease $\mathrm{SrtO}_{2} ; \%$ min } \\
\hline$>5 \%$ from baseline & $319[131-701]$ & 297 [207-895] & 0.717 \\
\hline$>10 \%$ from baseline & $119[29-358]$ & $119[63-502]$ & 0.805 \\
\hline$>15 \%$ from baseline & $46[10-157]$ & $54[20-312]$ & 0.761 \\
\hline $\begin{array}{l}\text { AUT of renal region specific tissue } \\
\text { deoxygenation; \% min }\end{array}$ & 720 [143-1293] & 443 [116-1507] & 0.532 \\
\hline \multicolumn{4}{|l|}{$\mathrm{SctO}_{2}$} \\
\hline Baseline; \% & $65(10)$ & $63(10)$ & 0.547 \\
\hline \multicolumn{4}{|l|}{ AUT absolute decrease $\mathrm{SctO}_{2} ; \%$ min } \\
\hline Below $90 \% \mathrm{SctO}_{2}$ & $5984(2219)$ & $7697(2020)$ & 0.051 \\
\hline Below $80 \% \mathrm{SctO}_{2}$ & $3710(2066)$ & $4989(2491)$ & 0.105 \\
\hline Below $70 \% \mathrm{SctO}_{2}$ & 1092 [445-3253] & $2520(1938)$ & 0.391 \\
\hline Below $60 \% \mathrm{SctO}_{2}$ & 146 [16-996] & 593 [109-1609] & 0.315 \\
\hline \multicolumn{4}{|l|}{ AUT relative decrease $\mathrm{SctO}_{2} ; \%$ min } \\
\hline$>5 \%$ from baseline & $177[60-576]$ & $289[158-711]$ & 0.571 \\
\hline$>10 \%$ from baseline & $57[13-255]$ & $147[41-287]$ & 0.391 \\
\hline$>15 \%$ from baseline & $21[3-90]$ & $67[9-92]$ & 0.375 \\
\hline \multicolumn{4}{|l|}{$\mathrm{SptO}_{2}$} \\
\hline Baseline; \% & $84(7)$ & $83(6)$ & 0.710 \\
\hline AUT $10 \%$ below baseline; $\%$ min & $36[0-313]$ & 215 [72-934] & $0.009^{*}$ \\
\hline
\end{tabular}

$\mathrm{SrtO}_{2}$ renal region tissue oxygenation, $\mathrm{SctO}_{2}$ cerebral tissue oxygenation, $\mathrm{SptO}_{2}$ peripheral tissue oxygenation, $A U T$ area under the threshold. Significance is indicated with $*$ for $\mathrm{p}<0.05$

\section{Discussion}

We did not observe an association between tissue oxygenation measured in the renal region or cerebral oxygenation and postoperative renal impairment in this small retrospective study. Surprisingly, a decrease in peripheral tissue oxygenation could predict postoperative renal impairment reasonably.

AKI frequently occurs after cardiac surgery $[1,2]$. In contrast to established AKI clasifications, postoperative renal impairment is more subtle, yet even these subtle changes in serum creatinine levels are associated with increased postoperative morbidity and mortality $[4,5]$. Therefore, it is important to assess the ability of different tools to predict impaired postoperative renal function and define patients at risk. Impaired postoperative renal function might be caused by ischemia or hypoxia during the intra-operative phase [17, 18]. Thus, it could be hypothesized that renal region tissue oxygenation monitoring by NIRS would be a useful predictor of postoperative renal impairment [10]. Several studies applying NIRS to the renal region in paediatric patients during cardiac surgery showed that decreased $\mathrm{SrtO}_{2}$ correlates with adverse outcomes and risk of renal impairment after cardiac surgery [11-15]. A possible explanation for the difference in predictive abilities between the paediatric and adult patients might be the positioning of the kidneys, which are closer to the skin in children. Another possible explanation is the different underlying morbidity in children. In a similar study of adult patients who underwent elective valve surgery, an absolute decrease in $\mathrm{SrtO}_{2}$ below 55\% correlated significantly with the risk of postoperative renal impairment [19]. However, an absolute decrease below 55\% hardly ever occurred in our population, which might indicate that the type of cardiac surgery could be an important factor in the development of postoperative renal impairment. An important difference between both studies is that we used renal depth $>5 \mathrm{~cm}$ as an exclusion criterion in our study instead of $>4 \mathrm{~cm}$ used in this study [19], which could also explain the different results. 
Table 3 Intra-operative oxygenation data of patients who underwent on-pump or off-pump CABG. Variables are mean (SD) or median [IQR]

\begin{tabular}{|c|c|c|c|}
\hline & Off-pump $(\mathrm{n}=22)$ & On-pump $(\mathrm{n}=19)$ & $P$ value \\
\hline \multicolumn{4}{|l|}{$\mathrm{SrtO}_{2}$} \\
\hline Baseline; $\%$ & $78(10)$ & $81(9)$ & 0.312 \\
\hline \multicolumn{4}{|l|}{ AUT absolute decrease $\mathrm{SrtO}_{2} ; \%$ min } \\
\hline Below $90 \% \mathrm{SrtO}_{2}$ & $2218[1302-3959]$ & $2591[1922-3583]$ & 0.480 \\
\hline Below $80 \% \mathrm{SrtO}_{2}$ & $752[125-1700]$ & 802 [389-1591] & 0.433 \\
\hline Below $70 \% \mathrm{SrtO}_{2}$ & $119[8-348]$ & $121[51-301]$ & 0.695 \\
\hline Below $60 \% \mathrm{SrtO}_{2}$ & $19[0-100]$ & $10[0-41]$ & 0.463 \\
\hline \multicolumn{4}{|l|}{ AUT relative decrease $\mathrm{SrtO}_{2} ; \% \min$} \\
\hline$>5 \%$ from baseline & $276[74-500]$ & $452[238-911]$ & 0.089 \\
\hline$>10 \%$ from baseline & 107 [14-213] & $166[67-502]$ & 0.182 \\
\hline$>15 \%$ from baseline & $28[10-123]$ & 77 [13-267] & 0.306 \\
\hline $\begin{array}{l}\text { AUT of renal region specific tissue deoxy- } \\
\text { genation; \% } \mathrm{min}\end{array}$ & $327[81-1044]$ & 1015 [443-1784] & $0.025^{*}$ \\
\hline \multicolumn{4}{|l|}{$\mathrm{SctO}_{2}$} \\
\hline Baseline; \% & $65(8)$ & $63(12)$ & 0.612 \\
\hline \multicolumn{4}{|l|}{ AUT absolute decrease $\mathrm{SctO}_{2} ; \%$ min } \\
\hline Below $90 \% \mathrm{SctO}_{2}$ & $6273(2524)$ & $6641(2545)$ & 0.645 \\
\hline Below $80 \% \mathrm{SctO}_{2}$ & $3940(2154)$ & $4184(2367)$ & 0.733 \\
\hline Below $70 \% \mathrm{SctO}_{2}$ & $1227[666-3261]$ & 1777 [420-3891] & 0.948 \\
\hline Below $60 \% \mathrm{SctO}_{2}$ & 159 [52-996] & 193 [12-1609] & 0.923 \\
\hline \multicolumn{4}{|l|}{ AUT relative decrease $\mathrm{SctO}_{2} ; \%$ min } \\
\hline$>5 \%$ from baseline & 191 [86-537] & $230[44-652]$ & 0.907 \\
\hline$>10 \%$ from baseline & $57[17-268]$ & $127[14-272]$ & 0.826 \\
\hline$>15 \%$ from baseline & $25[3-118]$ & $49[5-83]$ & 0.995 \\
\hline \multicolumn{4}{|l|}{$\mathrm{SptO}_{2}$} \\
\hline Baseline; \% & $85(5)$ & $82(8)$ & 0.180 \\
\hline AUT $10 \%$ below baseline; $\%$ min & 69 [1-363] & $68[5-498]$ & 0.854 \\
\hline
\end{tabular}

$\mathrm{SrtO}_{2}$ renal region tissue oxygenation, $\mathrm{SctO}_{2}$ cerebral tissue oxygenation, $\mathrm{SptO}_{2}$ peripheral tissue oxygenation, $A U T$ area under the threshold. Significance is indicated with $*$ for $\mathrm{p}<0.05$
In the past it has been suggested that cerebral oxygenation not only reflects oxygen status of the brain, but might also be an indication of oxygenation status in the entire body [20]. Cerebral oxygen saturation levels $<50 \%$ in cardiac surgery patients were found to be correlated with all-cause morbidity and mortality, in addition to cerebrovascular risk [21]. Therefore, we also assessed the predictive abilities of cerebral oxygenation. In our limited population we found no association between postoperative renal impairment and deoxygenation between absolute or relative thresholds of cerebral tissue deoxygenation. However, the 90\% AUT absolute decrease in $\mathrm{SctO}_{2}$ almost reached statistical significance $(p=0.051)$, when compared between patients with and without postoperative renal impairment (Table 2). Hence, it may be speculated that if a larger sample size was observed, this association would prove to be statistically significant. Current evidence for predicting AKI with cerebral oxygenation is inconsistent. In one study assessing cerebral oxygenation in 150 adult patients undergoing cardiac surgery under $\mathrm{CPB}$, cerebral oxygenation could not predict postoperative
AKI [22], confirming our results. On the other hand, in a study including 59 paediatric cardiac surgery procedures, cerebral oxygenation was found to be a good predictor of renal replacement therapy with an AUROC of 0.866 (95\% CI 0.770 to 0.961 ) [23]. One must note that this population was remarkably different regarding anatomy and type of surgery than the population we studied. However, in another study involving 45 adult patients undergoing cardiac surgery under CPB, an association was found between lower values of cerebral oxygenation and higher incidence of AKI (odds ratio, 0.667; 95\% confidence interval, 0.485-0.917; $\mathrm{p}=0.013$ ) [24]. Of note, both studies including adult patients were conducted solely in on-pump cardiac surgery and not in off-pump procedures.

In contrast to renal region and cerebral tissue oxygenation, the AUT of a decrease from baseline $>10 \%$ of peripheral tissue oxygenation at the thenar muscle did appear to be a reasonable predictor of postoperative renal impairment. This finding could be well explained by assuming that we did not actually measure renal tissue oxygenation, rather 
Table 4 Results of ROC analyses

\begin{tabular}{llll}
\hline & AUROC & $95 \%$ CI & P value \\
\hline SrtO $_{2}$ & & & \\
Baseline, $\%$ & 0.439 & 0.225 to 0.653 & 0.556 \\
AUT 90\% & 0.597 & 0.366 to 0.828 & 0.346 \\
AUT 80\% & 0.588 & 0.363 to 0.813 & 0.393 \\
AUT 70\% & 0.612 & 0.407 to 0.817 & 0.276 \\
AUT 60\% & 0.626 & 0.415 to 0.837 & 0.222 \\
AUT $>5 \%$ from baseline & 0.539 & 0.341 to 0.737 & 0.702 \\
AUT $>10 \%$ from baseline & 0.527 & 0.323 to 0.731 & 0.791 \\
AUT $>15 \%$ from baseline & 0.533 & 0.327 to 0.739 & 0.746 \\
Renal region specific tissue deoxygenation & & & \\
AUT of renal specific tissue deoxygenation & 0.433 & 0.232 to 0.634 & 0.517 \\
SctO 2 & & & \\
Baseline, $\%$ & 0.453 & 0.257 to 0.649 & 0.648 \\
AUT 90\% & 0.676 & 0.472 to 0.880 & 0.088 \\
AUT 80\% & 0.648 & 0.448 to 0.849 & 0.149 \\
AUT 70\% & 0.591 & 0.381 to 0.801 & 0.377 \\
AUT 60\% & 0.605 & 0.406 to 0.804 & 0.310 \\
AUT $>5 \%$ from baseline & 0.561 & 0.354 to 0.767 & 0.556 \\
AUT $>10 \%$ from baseline & 0.591 & 0.391 to 0.791 & 0.377 \\
AUT $>15 \%$ from baseline & 0.592 & 0.399 to 0.786 & 0.369 \\
SptO 2 & & & \\
Baseline, $\%$ & 0.442 & 0.257 to 0.628 & 0.576 \\
AUT $>10 \%$ from baseline & 0.767 & 0.619 to 0.914 & $0.010 *$ \\
Preoperative serum creatinine, $\mu$ mol $1^{-1}$ & 0.508 & 0.334 to 0.864 & 0.941 \\
\hline
\end{tabular}

$\mathrm{SrtO}_{2}$ renal region tissue oxygenation, $\mathrm{SctO}_{2}$ cerebral tissue oxygenation, $\mathrm{SptO}_{2}$ peripheral tissue oxygenation, AUT area under the threshold. Significance is indicated with $*$ for $\mathrm{p}<0.05$ than surrounding tissues as well. It is possible that peripheral tissue oxygenation provides a better indication of 'general' tissue oxygenation status. One of the aforementioned studies compared regional oxygen saturation in the thigh, forehead and abdomen also found that tissue oxygenation of the thigh was an independent risk factor for developing AKI [22], indeed suggesting that somatic oxygen saturation might provide a better indication of 'general' tissue oxygenation status. However, further research is needed to address this finding.

Since we studied a group of patients randomized to CABG performed with or without CPB, we were able to compare oxygenation data not only based on the development of renal impairment, but also between type of surgery. In our study we did not find any differences in renal region, cerebral and peripheral oxygenation between on-pump and off-pump CABG. However, the burden of renal region specific tissue deoxygenation was significantly higher in patients undergoing on-pump CABG. As mentioned before, CPB can cause disturbances in renal blood flow autoregulation and thus perfusion [8]. Additionally, the non-pulsatile flow usually used during CPB might hamper vascular responses to altered renal blood flow and consequently cause damage to renal tubules and glomeruli [25]. The literature contains conflicting data on the influence of surgical techniques on renal outcomes. Although a meta-analysis showed that offpump CABG (compared with on-pump CABG) was not associated with improved renal outcomes [26], a large RCT including 4,752 patients undergoing isolated CABG in 19 countries showed that the use of off-pump CABG reduced the risk of postoperative AKI compared to on-pump [27].

Lastly, postoperative renal impairment might be associated with a longer duration of surgery and a larger number of grafts created ( 2 grafts $(n=7), 3$ grafts $(n=20), 4$ grafts $(n=12), 5$ grafts $(n=2))$. The duration of surgery and the number of grafts were significantly correlated in our study $(r=0.330)$. No differences were found between the number of grafts and renal region and peripheral oxygenation, except for the difference in $\mathrm{AUT} \mathrm{SptO}_{2}>10 \%$ from baseline $(p=0.02)$, which increased with the number of grafts created. The duration of surgery as possible risk factor for renal impairment was already suggested previously [28].

In our study we defined baseline values of oxygenation as the mean of those recorded during the 5-min interval prior to incision. Post-induction baseline values might be a better representation of physiologic conditions during surgery 
than awake baseline values and might therefore be a more realistic basis on which to determine treatment goals [29]. It could be argued that the most substantial part of the damage to the kidneys will occur intraoperatively rather than during induction, and therefore deviations in oxygenation below a stable post-induction level height of oxygenation may be more relevant. In a study, already mentioned above, $\mathrm{SrtO}_{2}$ data was recorded from pre-induction until the end of procedure, and this data showed only minor differences in oxygenation between the pre- and post-induction periods [19]. Additionally, the $\mathrm{SrtO}_{2}$ data from this study showed that the most significant decreases in $\mathrm{SrtO}_{2}$ occurred during surgery and further that the extent of the $\mathrm{SrtO}_{2}$ decrease was related to the duration of surgery.

One of the limitations of this study is the small sample size, which was caused by the design of the original study (pilot study) and the necessity to exclude 18 patients due to missing data. Moreover, only a few patients developed postoperative renal impairment. Another limitation is that the depth of the kidney was not recorded systematically. Therefore, it may be possible that in some of the patients, oxygenation of more superficial tissue was measured instead of the renal tissue. This may partly be responsible for the lack of predictive abilities from renal region oxygenation. In the original study, patients undergoing low-risk cardiac surgery were included and therefore the results cannot be extrapolated to the entire population undergoing cardiac surgery. For future research it would be interesting to have larger sample sizes and to include patients undergoing different types of cardiac surgery and patients having comorbidities in order to verify the generalizability of these results, for which the current observations might serve as reference. It would also be interesting to include awake baseline values and expand the monitoring period to the postoperative phase.

In conclusion, we did not observe an association between tissue oxygenation measured in the renal region and cerebral oxygenation and postoperative renal impairment in this small retrospective study. Instead, peripheral tissue deoxygenation was able to predict postoperative renal impairment, suggesting that monitoring peripheral tissue oxygenation provides a better indication of 'general' tissue oxygenation status.

\section{Acknowledgements None.}

Funding No funding was received for conducting this study.

Data availability Data and materials are available upon request.

\section{Declarations}

Conflict of interest INdK, MP and JJV have no conflict of interest to declare. ARA is an editor of the British Journal of Anaesthesia. He reports reimbursement for consultancy work and paid phase 1 research by The Medicines Company (Parsippany, NJ, USA) and Rigel Inc
(San Francisco, CA); unrestricted research funding and reimbursenment for consultancy work from Carefusion (BD; Eysins, Switzerland) and Philips (Eindhoven, The Netherlands); receipt of consultancy fees from Janssen Pharma (Johnson and Johnson; Beerse, Belgium), Ever Pharma (Unterach am Attersee, Austria), PAION (Aachen, Germany) and Orion (Espoo, Finland). All payments made to institution. MAM received grants from AtriCure (Mason, Ohio, USA), Abbott Laboratories (Chicago, Illinois, USA), Edwards Lifesciences (Irvine, California, USA), Getinge Consultancy (Göteborg, Sweden), LivaNova PLC (London, United Kingdom) and Medtronic (Dublin, Ireland). TWLS received research grants and honoraria from Edwards Lifesciences (Irvine, CA, USA) and Masimo Inc. (Irvine, CA, USA) for consulting and lecturing and from Pulsion Medical Systems SE (Feldkirchen, Germany) for lecturing. TWLS is Editor-in-Chief of the Journal of Clinical Monitoring and Computing but had no role in the handling of this manuscript.

Ethical approval Ethical approval (Reference: METc2011/045) was obtained from the institutional ethical committee, Groningen, The Netherlands. The study was registered at ClinicalTrials.gov (NCT01347827).

Consent to participate Informed consent was obtained from all individual participants included in the study.

Open Access This article is licensed under a Creative Commons Attribution 4.0 International License, which permits use, sharing, adaptation, distribution and reproduction in any medium or format, as long as you give appropriate credit to the original author(s) and the source, provide a link to the Creative Commons licence, and indicate if changes were made. The images or other third party material in this article are included in the article's Creative Commons licence, unless indicated otherwise in a credit line to the material. If material is not included in the article's Creative Commons licence and your intended use is not permitted by statutory regulation or exceeds the permitted use, you will need to obtain permission directly from the copyright holder. To view a copy of this licence, visit http://creativecommons.org/licenses/by/4.0/.

\section{References}

1. Bastin A, Ostermann M, Slack A, Diller G, Finney S, Evans T. Acute kidney injury after cardiac surgery according to risk/injury/ failure/loss/end-stage, acute kidney injury network, and kidney disease: improving global outcomes classifications. J Crit Care. 2013;28:389-96.

2. Lagny M, Jouret F, Koch J, Blaffart F, Donneau A, Albert A, et al. Incidence and outcomes of Acute kidney injury after cardiac surgery using either criteria of the RIFLE classification. BMC Nephrol. 2015;16:76.

3. Kork F, Balzer F, Spies CDC, Wernecke KKD, Ginde AAA, Janowski J, et al. Minor postoperative increases of creatinine are associated with higher mortality and longer hospital length of stay in surgical patients. Anesthesiology. 2015;123:1301-11.

4. Lassnigg A, Schmid E, Hiesmayr M, Falk C, Druml W, Bauer $\mathrm{P}$, et al. Impact of minimal increases in serum creatinine on outcome in patients after cardiothoracic surgery: do we have to revise current definitions of acute renal failure? Crit Care Med. 2008;36:1129-37.

5. Bouma HR, Mungroop HE, de Geus AF, Huisman DD, Nijsten MWN, Mariani MA, et al. Acute kidney injury classification after cardiac valve operations. Ann Thorac Surg. 2018;106:92-8. 
6. Bernardi MH, Ristl R, Neugebauer T, Hiesmayr MJ, Druml W, Lassnigg A. Very early changes in serum creatinine are associated with 30-day mortality after cardiac surgery: A cohort study. Eur J Anaesthesiol. 2020;37:898-907.

7. Ishani A, Nelson D, Clothier B, Schult T, Nugent S, Greer N, et al. The magnitude of acute serum creatinine increase after cardiac surgery and the risk of chronic kidney disease, progression of kidney disease, and death. Arch Intern Med. 2011;171:226-33.

8. Sgouralis I, Evans R, Gardiner B, Smith J, Fry B, Layton A. Renal hemodynamics, function, and oxygenation during cardiac surgery performed on cardiopulmonary bypass: a modeling study. Physiol Rep. 2015;3:e12260.

9. Grosenick D, Cantow K, Arakelyan K, Wabnitz H, Flemming B, Skalweit A, et al. Detailing renal hemodynamics and oxygenation in rats by a combined near-infrared spectroscopy and invasive probe approach. Biomed Opt Express. 2015;6:309-23.

10. Tholén M, Ricksten SE, Lannemyr L. Renal near-infrared spectroscopy for assessment of renal oxygenation in adults undergoing cardiac surgery: a method validation study. J Cardiothorac Vasc Anesth. 2020;34:3300-5.

11. Gist K, Kaufman J, da Cruz E, Friesen R, Crumback S, Linders M, et al. A decline in intraoperative renal near-infrared spectroscopy is associated with adverse outcomes in children following cardiac surgery. Pediatr Crit Care Med. 2016;17:342-9.

12. Owens G, King K, Gurney J, Charpie J. Low renal oximetry correlates with acute kidney injury after infant cardiac surgery. Pediatr Cardiol. 2011;32:183-8.

13. Hazle M, Gajarski R, Aiyagari R, Yu S, Abraham A, Donohue $\mathrm{J}$, et al. Urinary biomarkers and renal near-infrared spectroscopy predict intensive care unit outcomes after cardiac surgery in infants younger than 6 months of age. J Thorac Cardiovasc Surg. 2013;146(861-867):e1.

14. Hoffman G, Ghanayem N, Scott J, Tweddell J, Mitchell M, Mussato K. Postoperative cerebral and somatic near-infrared spectroscopy saturations and outcome in hypoplastic left heart syndrome. Ann Thorac Surg. 2017;103:1527-35.

15. Ruf B, Bonelli V, Balling G, Hörer J, Nagdyman N, Braun SL, et al. Intraoperative renal near-infrared spectroscopy indicates developing acute kidney injury in infants undergoing cardiac surgery with cardiopulmonary bypass: a case-control study. Crit Care. 2015;19:27.

16. Kok W, van Harten A, Koene B, Mariani M, Koerts J, Tucha O, et al. A pilot study of cerebral tissue oxygenation and postoperative cognitive dysfunction among patients undergoing coronary artery bypass grafting randomised to surgery with or without cardiopulmonary bypass. Anaesthesia. 2014;69:613-22.

17. Ranucci M, Romitti F, Isgrò G, Cotza M, Brozzi S, Boncilli A, et al. Oxygen delivery during cardiopulmonary bypass and acute renal failure after coronary operations. Ann Thorac Surg. 2005;80:2213-20.
18. Singh P, Ricksten S, Bragadottir G, Redfors B, Nordquist L. Renal oxygenation and haemodynamics in acute kidney injury and chronic kidney disease. Clin Exp Pharmacol Physiol. 2013;40:138-47.

19. Choi D, Kim W, Chin J, Lee E, Don Hahm K, Yeon Sim J, et al. Intraoperative renal regional oxygen desaturation can be a predictor for acute kidney injury after cardiac surgery. J Cardiothorac Vasc Anesth. 2014;28:564-71.

20. Murkin J. Cerebral oximetry: monitoring the brain as the index organ. Anesthesiology. 2011;114:12-3.

21. Heringlake M, Garbers C, Käbler JH, Anderson I, Heinze H, Schön J, et al. Preoperative cerebral oxygen saturation and clinical outcomes in cardiac surgery. Anesthesiology. 2011;114:58-69.

22. Sakaki K, Kitamura T, Kohira S, Torii S, Mishima T, Hanayama $\mathrm{N}$, et al. Regional Thigh Tissue Oxygen Saturation During Cardiopulmonary Bypass Predicts Acute Kidney Injury After Cardiac Surgery [published online ahead of print, 2020 May 24]. J Artif Organs. 2020;23:315-20.

23. Saito J, Takekawa D, Kawaguchi J, Suganuma T, Konno M, Noguchi $\mathrm{S}$, et al. Preoperative cerebral and renal oxygen saturation and clinical outcomes in pediatric patients with congenital heart disease. J Clin Monit Comput. 2019;33:1015-22.

24. Balci C, Haftaci E, Kunt AT. Use of cerebral oxygen saturation and hemoglobin concentration to predict acute kidney injury after cardiac surgery. J Int Med Res. 2018;46:1130-7.

25. Salameh A, Kühne L, Grassl M, Gerdom M, Von Salisch S, Vollroth M, et al. Protective effects of pulsatile flow during cardiopulmonary bypass. Ann Thorac Surg. 2015;99:192-9.

26. Cheng DDC, Bainbridge D, Martin JJE, Novick RJR. Does offpump coronary artery bypass reduce mortality, morbidity, and resource utilization when compared with conventional coronary artery bypass? A meta-analysis of randomized trials. Anesthesiology. 2005;102:188-203.

27. Garg AX, Devereaux PJ, Yusuf S, Cuerden MS, Parikh CR, Coca $\mathrm{SG}$, et al. Kidney function after off-pump or on-pump coronary artery bypass graft surgery: a randomized clinical trial. JAMA. 2014;311:2191-8.

28. Borthwick E, Ferguson A. Perioperative acute kidney injury: risk factors, recognition, management, and outcomes. BMJ. 2010;341:c3365.

29. Modestini M, Hoffmann L, Niezen C, Armocida B, Vos JJ, Scheeren TWL. Cerebral oxygenation during pediatric congenital cardiac surgery and its association with outcome: a retrospective observational study. Can J Anesth. 2020;67:1170-81.

Publisher's Note Springer Nature remains neutral with regard to jurisdictional claims in published maps and institutional affiliations. 